\title{
Research on Determining the Index Weight of DS/FH TT\&C Anti-jamming Effectiveness Evaluation based on Entropy Method
}

\author{
Hong Ma ${ }^{a}$, Shaojie Wei ${ }^{b}$, Guoling Qin ${ }^{\mathrm{c}}$ \\ Equipment Academy Beijing, 101416, China \\ amahong_0108@163.com, bsevenwsj@126.com, 6554432823@qq.com
}

Keywords: DS/FH TT\&C; effectiveness evaluation; index weight.

\begin{abstract}
Anti-jamming effectiveness evaluation refers to measure and evaluate the DS/FH TT\&C performance which is affected by the jamming environment, is an important way to assess anti-jamming performance and optimize TT\&C system. Scientific and completed index weight is the key on reasonable and believable of assessment result. An improved algorithm is proposed to solve the problem that weight change is big when entropy tends to 1 and weight is little when entropy tends to 0 , objective weight is settled by studying the change under different jamming signal ratio.
\end{abstract}

\section{Introduction}

$\mathrm{DS} / \mathrm{FH}$ (direct sequence/ frequency hopping) TT\&C(telemetry, tracking and control) system which is a new generation of telemetry, tracking and control system is researched to satisfy the requirement of aerospace TT\&C in strong electromagnetic interference environment. The system has the advantage of anti-broadband, narrowband and anti-multipath in DS (direct sequence), anti-tracking, anti-single tone interference in FH (frequency hopping) ${ }^{[1]}$. This system has a strong ability of anti jamming and the anti interception performance is also good.

Developing DS/FH control system anti jamming effectiveness evaluation, has great significance in analysis of the ability to adapt strong interference environment and objective and scientific evaluation of anti-jamming performance etc in different DS/FH TT\&C. Index weight is the foundation of effectiveness evaluation. Scientific and reasonable index weight is the base of reasonable and credible evaluation results. Currently, there are many relevant index weighting method, including AHP ${ }^{[1]}$, grey correlation ${ }^{[2-3]}$, entropy method ${ }^{[4]}$ etc.

Entropy method realize weighting by extracting the index change of information entropy. The method is not affected by the subjective intention and it can response the relation between index change and index weight. This method is a kind of objective weighting method. The conversion between entropy and entropy weight is the key of entropy method and it determines the credible degree of index weight. This paper put forward an improved entropy method which is suitable for the small data with large fluctuations.

\section{Analysis and improvement of entropy method}

The basic steps of entropy method. Suppose that there are $n$ objects of evaluation and $m$ index, the data matrix $X=\left\{x_{i j}\right\}_{n \times m} x_{i j}$ is the evaluation value that object $i$ evaluate index $j$. The basic idea of entropy method is that if the change $x_{i j}$ is bigger, the weight of index $j$.is bigger; if $x_{i j}$ is constant, the index in the evaluation does not work and the weight is 0 .

The basic steps of entropy method are below:

Normalizing the index. Calculate the proportion $p\left(x_{i j}\right)$ of it's that object $i$ evaluate index $j$.

$$
p\left(x_{i j}\right)=\frac{x_{i j}}{\sum_{i=1}^{n} x_{i j}}(i=1,2, \ldots, n ; j=1,2, \ldots, m)
$$

Calculate the information entropy $e_{j}$ of $x_{i j}$. 
(2)

$e_{j}=-\frac{1}{\ln (n)} \sum_{i=1}^{n} p\left(x_{i j}\right) \ln \left(p\left(x_{i j}\right)\right)$

Calculate the differential coefficient of index $j$.

$h_{j}=1-e_{j}$

The $h_{j}$ is bigger, the influence of the index to the evaluate results is greater.

Normalize $h_{j}$ :

$$
w_{j}=\frac{h_{j}}{\sum_{j=1}^{n} h_{j}}
$$

In a word, entropy method determine the index weight through analysis and comparison of indexes change. It is a kind of objective weighting method.

Improvement of entropy method. When the information entropy $e_{j}$ of entropy method approaches 1, even small differences between each other will also cause multiplied change of different index weight. For example, when the entropy is [0.999, 0.998, 0.997, 0.996, 0.995 ], the weight of index by calculating is [0.067, 0.133, 0.2, 0.267, 0.333]. The difference of the entropy is small, but the difference of index weight is huge. This is because of that entropy weight method is too sensitive when $e_{j} \rightarrow 1$. And if the index entropy is 1 , then the corresponding weight is 0 . This is very unreasonable. In order to solve the problems discussed above, many scholars try to optimize and improve the entropy method from different points of view ${ }^{[5-6]}$.

This paper puts forward a new method. by improving the computation formula of coefficient of variation. The method has the advantages of simple operation and easy to understand. It can better solve the problems discussed above.

Suppose the information entropy $e_{j}=a_{j}, a_{j} \in(0,1], j=1,2, \ldots n, a=\min _{j=1,2 \ldots n}\left(a_{j}\right)$.

Improve the difference coefficient:

$h_{j}=1+a-e_{j}$

Now proves the rationality of the formula (5).

First demonstrate the relationship between index weight $w_{j}$ and the difference coefficient.

Suppose a series of index entropy $e_{j}(j=1,2, \ldots, m)$ is known.

$$
w_{j}=\frac{h_{j}}{\sum_{j=1}^{n} h_{j}}=\frac{1+a-e_{j}}{\sum_{j=1}^{n}\left(1+a-e_{j}\right)}
$$

Because $\sum_{j=1}^{n}\left(1+a-e_{j}\right)$ is a constant value, $w_{j}$ decreases when $h_{j}$ increases. It satisfies the definition of the relation between entropy and the difference coefficient.

For two entropy index ( $e_{m}$ and $e_{p}$ ) with small difference, the relationship between the corresponding weights is shown in formula (7). $\left(e_{p}-e_{m}\right) /\left(1+a-e_{p}\right)$ Is a small variable, so $\left(w_{m}\right) /\left(w_{p}\right) \approx 1$, the difference between the corresponding weights is not large. Thus, when the index entropy change slightly, the improved method will not appear large fluctuation of the index weight.

$$
\frac{w_{m}}{w_{p}}=\frac{1+a-e_{m}}{\sum_{m=1}^{n}\left(1+a-e_{m}\right)} / \frac{1+a-e_{p}}{\sum_{p=1}^{n}\left(1+a-e_{p}\right)}=\frac{1+a-e_{m}}{1+a-e_{p}}=1+\frac{e_{p}-e_{m}}{1+a-e_{p}}
$$

For two entropy index ( $e_{m}$ and $e_{p}$ ) with large difference, the relationship between the corresponding weights is shown in formula (8). The improved method can reduce the gap of the different index weight. 


$$
\frac{w_{m}}{w_{p}}=\frac{1+a-e_{m}}{\sum_{m=1}^{n}\left(1+a-e_{m}\right)} / \frac{1+a-e_{p}}{\sum_{p=1}^{n}\left(1+a-e_{p}\right)}=\frac{1+a-e_{m}}{1+a-e_{p}}>\frac{1-e_{m}}{1-e_{p}}
$$

For the case that the index entropy is 1 , the difference coefficient which is calculated by the formula (5) is not 0 ,

Using 3 different indexes as examples, the comparison and analysis of the index weight of three index weighting method ( the entropy method, the method in literature [6] and the improved method ) are shown in table 1.

Table 1 the comparison and analysis of the index weight

\begin{tabular}{|c|c|c|c|}
\hline $\begin{array}{c}\text { index } \\
\text { weight }\end{array}$ & entropy method & method in literature [6] & improved method \\
\hline$[0.999$ & {$[0.1667$} & {$[0.3333$} & {$[0.3333$} \\
0.998 & 0.3333 & 0.3333 & 0.3333 \\
$0.997]$ & $0.5]$ & $0.3334]$ & $0.3334]$ \\
\hline$[[0.9$ & {$[0.1667$} & {$[0.2963$} & {$[0.2963$} \\
0.5 & 0.3333 & 0.3333 & 0.3333 \\
$0.1]$ & $0.3750]$ & $0.3889]$ & $0.3704]$ \\
\hline$[0.0003$ & {$[0.3330$} & {$[0.333$} & {$[0.3333$} \\
0.0002 & 0.3333 & 0.3333 & 0.3333 \\
$0.0001]$ & $0.337]$ & $0.3334]$ & $0.3334]$ \\
\hline
\end{tabular}

The method basically overcomes the defects of great range change of the index weight. When the entropy value is close to 0.5 or close to 0 , the index weight of entropy method and improved method is almost same. Through the above analysis, the rationality of the improved algorithm is verified.

\section{Determining the index weight based on entropy method}

From the view of system, DS/FH TT\&C system mainly works in the capturing stage and tracking stage. Through the analysis and research of literature ${ }^{[7-9]}$, the system of DS/FH TT\&C anti-jamming effectiveness evaluation is constructed as shown in Fig. 2.

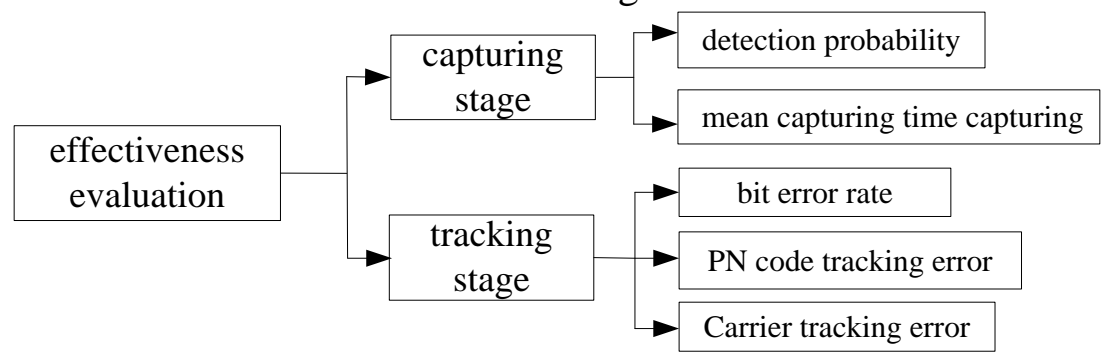

Fig.2 the system of DS/FH TT\&C anti-jamming effectiveness evaluation

The system of DS/FH TT\&C mainly faces the influence of various kinds of jamming, including wide-band jamming, narrow-band jamming, single-ton jamming, multi-tone jamming, pulse jamming, BPSK jamming and chirp jamming. Simulation analysis of index change with the JSR (jamming to signal ratio) range $25 d B-65 d B$.are done and improved entropy method are used to determine the weight of index. The changes of index in different JSR is shown in Fig.3.

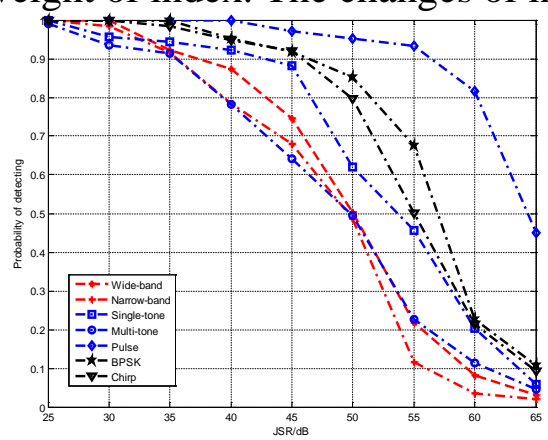

(a) Detection probability

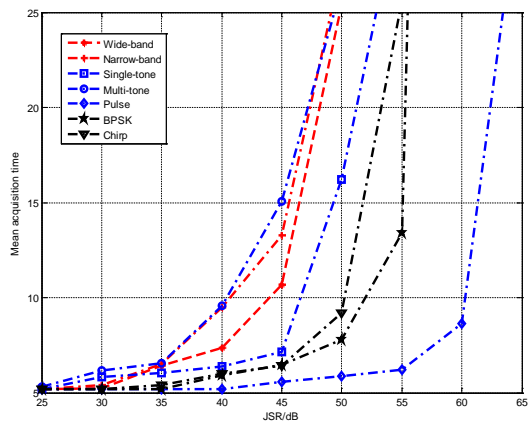

(b) mean capturing capturing time 


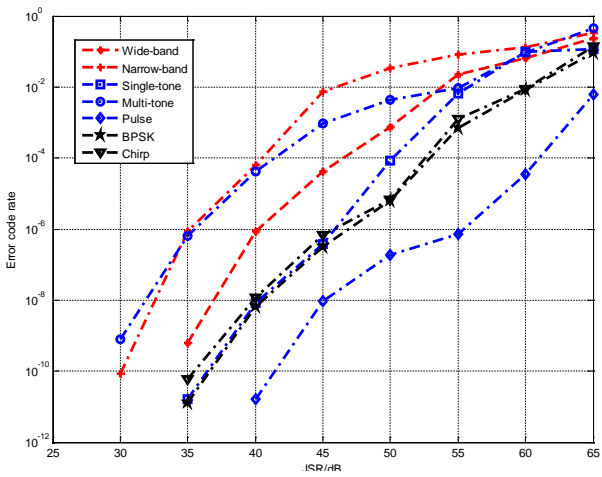

(c) Bit error rate

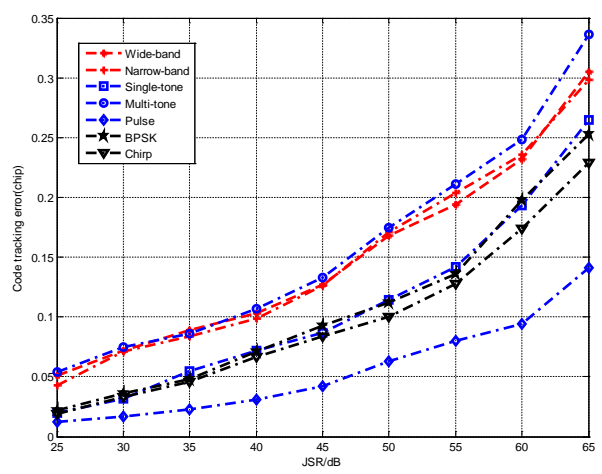

(d) PN code tracking error

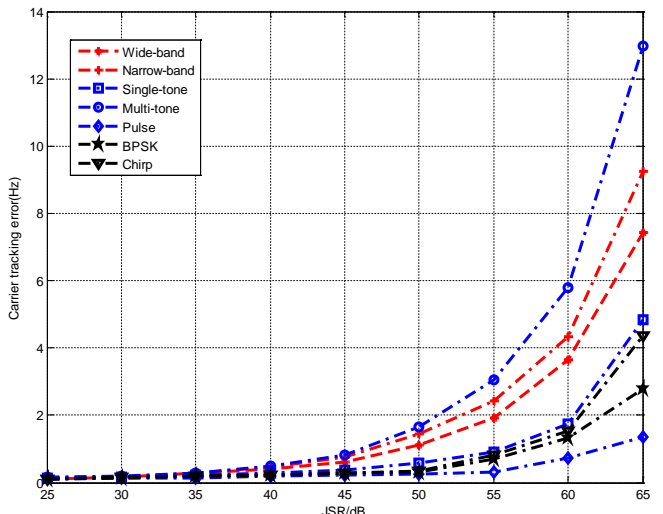

(e) Carrier tracking error

Fig. 3 the changes of index in different JSR

The steps of the improved entropy method are same as entropy method. Only adding a minimum entropy in the solution of difference coefficient. The index weight and variance of two different methods are shown in Fig.4.

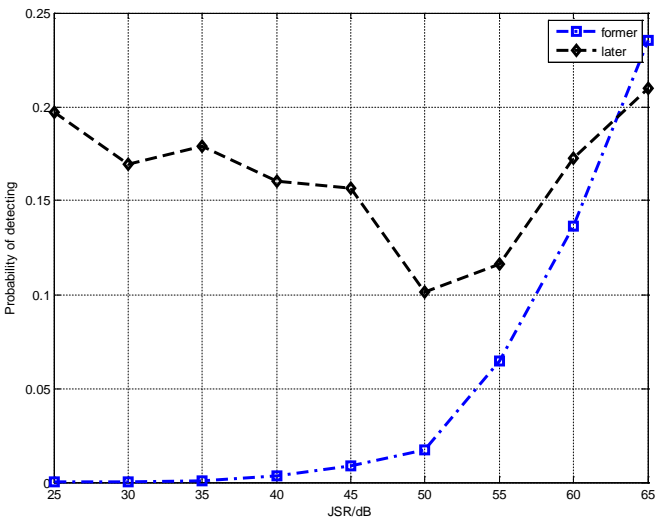

(a) Detection probability

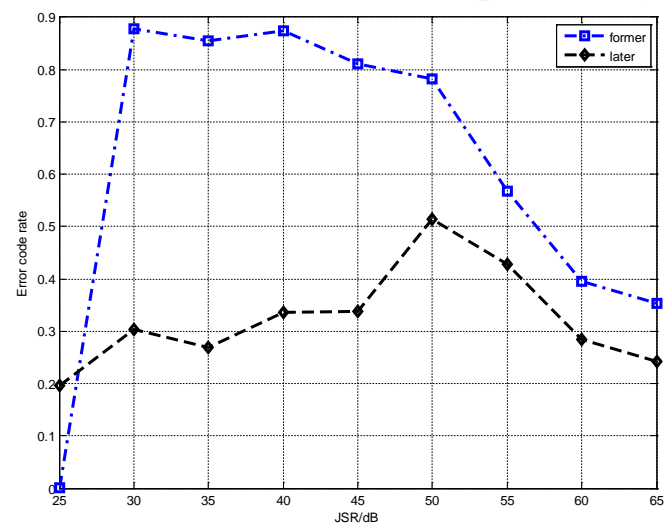

(c) Bit error rate

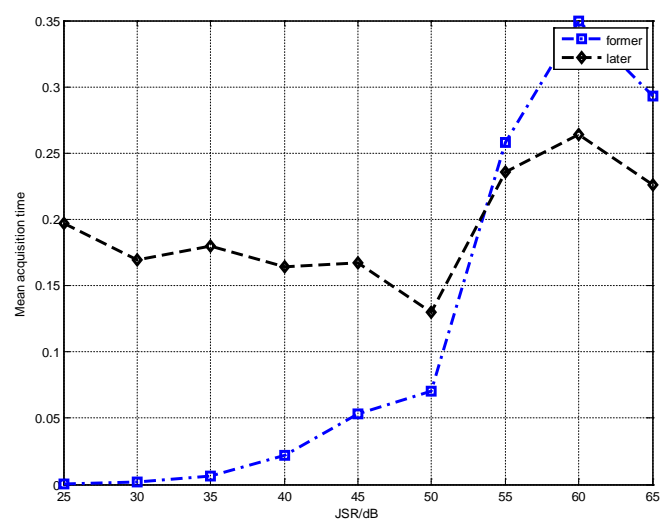

(b) mean capturing capturing time

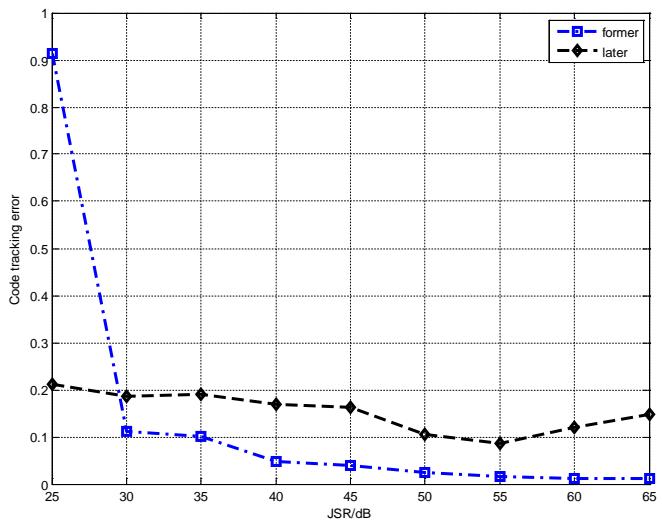

(d) PN code tracking error 


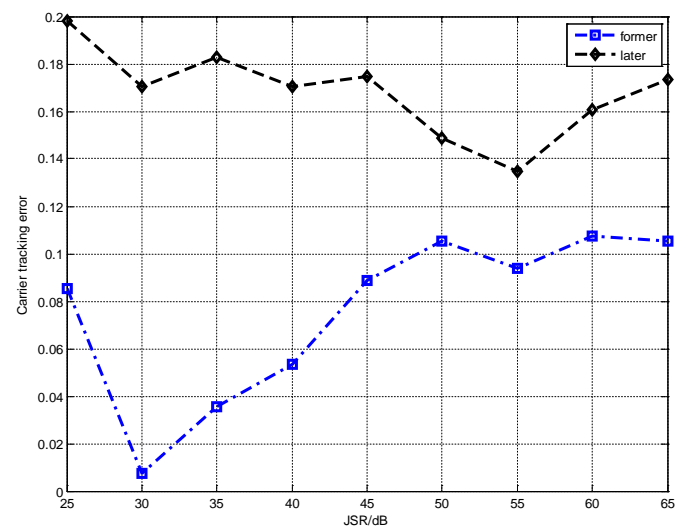

(e) Carrier tracking error

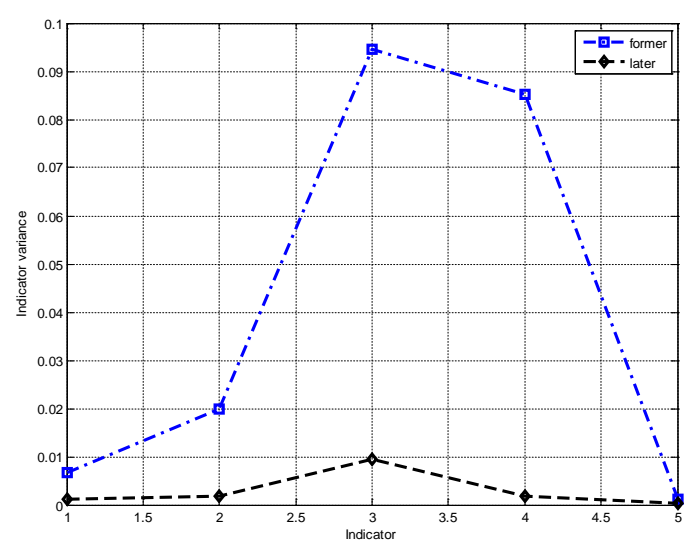

(f) variance

Fig.4 the index weight and variance of two different methods

\section{Summary}

From Fig. 4, we can get the following conclusion.

The improved method can reduce the gap of the different index weight.

The improved method overcome the small situation of index weight.

The improved method improve the relative size of the index weights, and avoid the appearance of mini or max weight.

The improved method solves the problems which entropy method has, and reduces the sensitivity for weak signal, creating favorable conditions for the DS/FH TT\&C system anti-jamming effectiveness evaluation.

\section{References}

[1] Meng Shengyun. Key technology research on Signal process of DS/FH hybrid spread spectrum in the TT\&C [D] Equipment Academy. 2012

[2] Hao Jianhua, XI Youyou, CHENG Naiping. Journal of Spacecraft TT\&C Technology, 2013, 32(2):106-110.

[3] Yan Junling, Du Xiaojia, Gao Lijuan. Hosp Admin J Chin PLA, 2013, 20(1): 30-32.

[4] Lian Shiwei, Li Xiuhe, Shen Yang. Electronic Information Warfare Technology, 2011, 26(6): 62-67.

[5] Zhang Jie, Tang Hong, Su Kai. Research on Effectiveness Evaluation Method [M]. Beijing: Nation Defend Industry Press, 2009.

[6] Zhou Huicheng, Zhang Gaihong, Wang Guoli. SHUILI XUEBAO, 2007, 38(1): 100-106.

[7] LI Yingghai, Zhou Jianzhong. Water Resources and Power, 2010, 28(6):32-35.

[8] Tian Ricai. Spread Communication [M]. Beijing: Tsinghua Press, 2012.

[9] Yang Xiaolong. DS/FH TT\&C Anti-jamming performance test and evaluate method research.

[D] Equipment Academy. 2014

[10] Xi Youyou, Hao Jianhua, Cheng Naiping. Telecommunications Science, 2. 